Figure 3 Relationship between the difference between the apnoea index assessed with the Autoset and by polysomnography (PSG) and the percentage time of nasal ventilation below 5 arbitrary units (range 0-20).

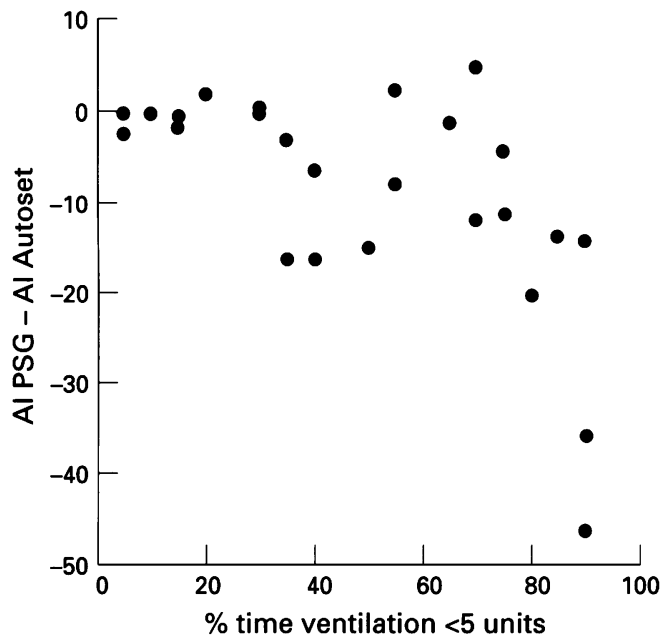

ticularly important to examine the raw data to assess the adequacy of the flow signal. A very low value for nasal ventilation on the report printout, for example, raises the suspicion of poor signal quality. Figures 1 and 2 show two outliers. Figure 3 shows that in both cases nasal ventilation was very low ( $<5$ arbitrary units) for $>90 \%$ of the study time (one outlier represents the patient after uvulopalatopharyngoplasty, one a patient with uncomplicated sleep apnoea). These points stress the importance of considering the complete data set of the report and not just the final number for the AI.

The Autoset is not designed to replace full diagnostic or therapeutic evaluation, but to provide adequate, although in some cases possibly provisional, care for the typical patient with sleep apnoea. The sensitivity and specificity of the method were therefore calculated for an AHI-PSG of $>20$, a level of respiratory disturbance that would warrant treatment in most sleep centres. ${ }^{1011}$ The sensitivity of $82 \%$ and the specificity of $90 \%$ show that a reasonable number of patients can be managed by this strategy as a first step, and that the Autoset did better in these patients than oximetry alone ( $76 \%$ and $69 \%$, respectively). Some caution is needed with the values for sensitivity and specificity because of the relatively small numbers of patients studied. Furthermore, these values also indicate that, in patients with a clinical suspicion of sleep apnoea but negative Autoset results, or positive Autoset results but unsatisfactory response to treatment, or whenever the results are ambiguous, full overnight polysomnography must be performed.

1 Partinen M. Epidemiology of sleep disorders. In: Kryger MH, Roth T, Dement WC, eds. Principles and practice of sleep medicine. 2nd ed. Philadelphia: WB Saunders, 1994 437-52.

2 Young T, Palta M, Dempsey J, Skatrud J, Weber S, Badr S. The occurrence of sleep-disordered breathing among middle-aged adults. $N$ Engl $\mathcal{F}$ Med 1993;328:1230-5.

3 Sullivan CE, Issa FG, Berthon-Jones M, Eves L. Reversal of obstructive sleep apnea by continuous positive airway pressure applied through the nares. Lancet 1981; arwa

4 pressure applied through the nares. Lancet 1981;1:862-5. agement of obstructive sleep apnoea/hypopnoea synagement of obstructive sleep
drome. Lancet 1994;344:656-60.

5 Miles LE, Buschek GD, McClintock DP, Miles SC, Narvios LR, Wang YX. Development and application of automatic nasal CPAP calibration procedures for use in the unsupervised home environment. Sleep 1993;16:S118-19.

6 Chediak AD, Lipson E, Cuneyt Demirozu M, Kiel M. The second generation of nasal continuous positive airway pressure devices. Are they created equal? Sleep 1993;16: 662-7.

7 Berthon-Jones M. Feasibility of a self-setting CPAP machine. Sleep 1993;16:S120-3.

8 Condos R, Norman RG, Krishnasamy I, Peduzzi N, Goldring RM, Rapport DM. Flow limitation as a noninvasive assessment of residual upper airway resistance during continuous positive airway pressure therapy of obstructive sleep apnea. Am F Respir Crit Care Med 1994;150:475-80

9 Gould GA, Whyte KF, Rhind GB, Airlie MAA, Catteral JR, Shapiro CM, et al. The sleep hypopnea syndrome. Am Rev Respir Dis 1988;137:895-8.

10 Kryger MH. Management of obstructive sleep apnea. Clin Chest Med 1992;13:481-92.

11 Phillipson EA. Sleep apnea, a major public health problem. $N$ Engl f Med 1993;328:1271-3.

\title{
Comparison of polysomnography with ResCare Autoset in the diagnosis of the sleep apnoea/hypopnoea syndrome
}

\author{
Pauline A Bradley, Ian L Mortimore, Neil J Douglas
}

\footnotetext{
Respiratory Medicine Unit, Department of Medicine, Royal Infirmary, Edinburgh EH3 9YW, UK P A Bradley I L Mortimore N J Douglas

Correspondence to: Professor N J Douglas.

Received 23 February 1995 Returned to authors 5 June 1995 Revised version received 20 July 1995 Accepted for publication 24 July 1995
}

\author{
Abstract \\ Background - Increasing referral num- \\ bers make the development of simplified \\ accurate methods of diagnosing the sleep \\ apnoea/hypopnoea syndrome highly de- \\ sirable. The accuracy of one such system - \\ the ResCare Autoset - has been examined. \\ Methods - Thirty one consecutive patients \\ assessed by polysomnography had sim- \\ ultaneous monitoring of their respiratory \\ pattern using the Autoset system. The \\ Autoset detects episodes of flattening of
}

the flow/time profile using nasal cannulae. Results - There was a good correlation $(r=0.85)$ between the number of apnoeas + hypopnoeas/hour in bed recorded using polysomnography and the Autoset system. The median difference in such events was $3 \cdot 1 \quad(95 \%$ confidence interval 8.4 to $-1 \cdot 6$ )/hour in bed. In two patients the Autoset scored 70 apnoeas + hypopnoeas/hour in bed compared with 34 apnoeas + hypopnoeas with 35 arousals/ hour in bed by polysomnography; how- 
ever, this did not alter the diagnostic category of either patient. Autoset gave a sensitivity of $100 \%$, specificity of $92 \%$, positive predictive value of $92 \%$, and negative predictive value of $100 \%$, which was better than oximetry alone. A sleep study using the Autoset system costs $£ 14$ compared with $£ 126$ for polysomnography. Conclusions - The Autoset is clinically useful for diagnosing the sleep apnoeal hypopnoea syndrome.

(Thorax 1995;50:1201-1203)

Keywords: polysomnography, sleep apnoea/hypopnoea, Autoset.

The sleep apnoea/hypopnoea syndrome (SAHS) may occur in $2-4 \%$ of middle aged men and $1-2 \%$ of middle aged women, ${ }^{12}$ indicating a large pool of as yet undiagnosed sufferers. Sleep laboratories all around the world are being overloaded by rapidly increasing referral rates. It is important that patients with this condition are diagnosed so that they can receive treatment, thus avoiding the sleepiness, impaired daytime cognitive function, ${ }^{34}$ increased road traffic accidents, ${ }^{56}$ and increased cardiovascular and cerebrovascular morbidity and mortality. ${ }^{78}$

There is therefore a need to develop simplified diagnostic techniques to allow hospitals without specialist sleep laboratories to diagnose and treat such patients. Screening for SAHS using oximetry alone gives false negative results in about one third of cases. ${ }^{9}$ It has been suggested that limited sleep study equipment should be based not on oximetry but on devices to detect the presence of apnoeas or hypopnoeas from the respiratory signal. ${ }^{9}$

The ResCare Autoset System ${ }^{10}$ was originally developed to titrate the required level of CPAP pressure automatically. It has been modified so that it can be used in the diagnostic mode by examining respiratory flow/time profiles and thus can theoretically detect, not only apnoeas and hypopnoeas, but also episodes of flow limitation ${ }^{11}$ when the flow/time curve is truncated as will occur in partial collapse of the upper airways. This device is simple to use and we have assessed its diagnostic value in patients undergoing simultaneous polysomnography.

Relationship between Autoset and

polysomnography results on (A) scatter plot and $(B)$ plot of difference against mean.

\section{Methods}

Thirty one consecutive patients (26 men) of mean (SE) age 46 (2), mean (SE) body mass index 30 (1) $\mathrm{kg} / \mathrm{m}^{2}$, and mean (SE) Epworth sleepiness score ${ }^{12}$ of 12 (1) were assessed using polysomnography ${ }^{9}$ and simultaneous monitoring by the Autoset (ResCare UK Ltd, Oxford, UK). The Autoset measures pressure in the anterior nares using nasal cannulae connected to a pressure manometer. Anterior nares pressure reflects flow and the flow/time profile is examined using software (ResCare Autoset version 2.0) in a $486 \mathrm{PC}$ to allow detection of apnoeas or hypopnoeas when the inspiratory flow/time profile is flattened. Software version 2.0 does not differentiate between apnoeas and hypopnoeas (both taken as more than $50 \%$ reduction in flow for at least 10 seconds) in analysis or printout of data, and we have therefore referred to this as the Autoset "apnoea" index. The Autoset system can also be connected to an external oximeter and a Biox 3700 (Ohmeda, Essex, UK) was used in this study.

Polysomnographic variables were scored using our standard criteria for sleep and respiratory signals ${ }^{9}$ and microarousals.

\section{DATA ANALYSIS}

As the Autoset device does not record sleep, the frequency of apnoeas and hypopnoeas (Autoset "apnoea" index) obtained with the Autoset was compared with the number of apnoeas and hypopnoeas which occurred either during sleep or during wakefulness on polysomnography. ${ }^{9}$ Polysomnography was compared with the Autoset using the Wilcoxon rank test, Bland and Altman plots, ${ }^{13}$ and correlation analysis. The results are reported as mean (SE). One observer (NJD) assessed the diagnostic category for each subject based on visual examination of overnight oximetry without reference to Autoset or polysomnography data. Oximetry recordings were categorised as definitely indicative of sleep apnoea/hypopnoea or other.

\section{Results}

On polysomnography the patients had a mean (SE) of 25 (4) apnoeas + hypopnoeas/hour

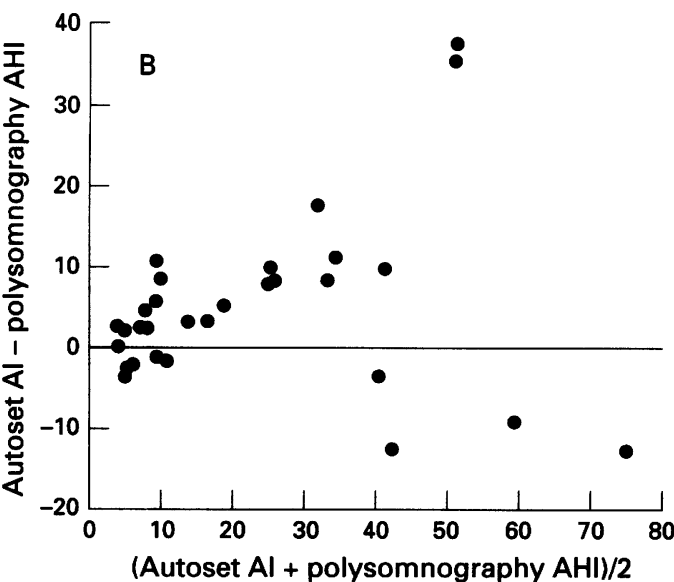

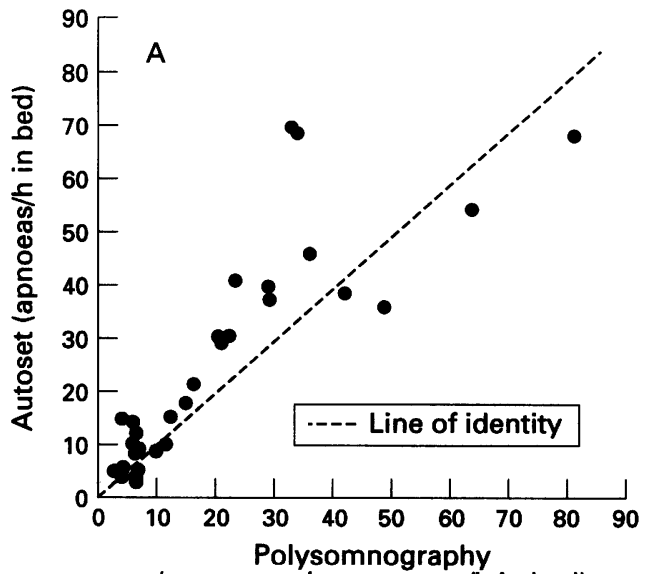

(apnoeas + hypopnoeas/h in bed) 
slept. The number of "apnoeas"/hour in bed obtained by the Autoset was significantly correlated $(r=0.85 ; \mathrm{p}<0.001)$ with the number of apnoeas + hypopnoeas/hour in bed obtained on polysomnography (figure). The Autoset tended to overscore "apnoeas", median difference $3 \cdot 1$ (95\% confidence interval 8.4 to $-1 \cdot 6$ )/hour in bed, although there was not a statistically significant difference in the number of events scored by the two techniques $(p>0.05)$. Two of the patients had much higher "apnoea" frequencies with the Autoset (69 and 70, respectively) than with polysomnography ( 34 and 33 apnoeas + hypopnoeas/hour in bed, respectively). These two subjects had 32 and 37 arousals/hour in bed. Examination of the flow signal in these subjects did not show evidence of sustained periods of mouth breathing or dislodgement of nasal cannulae.

If one uses a threshold for disease of $\geqslant 15$ apnoeas + hypopnoeas/hour of sleep, ${ }^{9}$ the Autoset gave a sensitivity of $100 \%$, specificity of $92 \%$, the positive predictive value was $92 \%$, and the negative predictive value $100 \%$. Oximetry alone gave a sensitivity of $75 \%$, specificity of $85 \%$, positive predictive value $75 \%$, and negative predictive value $89 \%$. Below 15 apnoeas and hypopnoeas/hour there was poor agreement between the Autoset and polysomnography; however, the diagnostic category of the subjects was not affected.

The cost of consumables for the Autoset system was $£ 2$ per subject (for nasal cannulae) compared with $£ 16$ per subject with polysomnography. The average time to set up the Autoset system was five minutes compared with 60 minutes for polysomnography. Our night staff did not need to adjust the Autoset system thereafter, whereas polysomnography needs constant attention. Thus, the total recurrent cost of an Autoset study was $£ 7$ per study compared with $f 104$ per study for polysomnography (which includes technician costs estimated at $£ 5$ for Autoset and $£ 50$ for polysomnography). The Autoset costs approximately $£ 10000$ plus VAT and the polysomnography system $£ 22000$. Thus, allowing for five year writedown of capital and 200 studies being performed/bed/year, the cost per study is $£ 14$ for the Autoset compared with $£ 126$ for polysomnography. This analysis excludes the capital cost of the bed used identical for both techniques.

\section{Discussion}

The Autoset system was cheap and easy to use and produced data with sufficient accuracy to allow patients with $>20$ apnoeas + hypopnoeas/ hour of sleep to be confidently diagnosed as having the sleep apnoea/hypopnoea syndrome. However, there was a considerable difference between the results with polysomnography and the Autoset in two patients. We believe that this is due to the Autoset diagnosing "apnoeas" when nasal airflow was continuing, albeit with minor nasal flow limitation, ${ }^{11}$ or possibly due to mouth breathing and/or dislodging of nasal cannulae, although we did not notice problems with cannulae positioning. The evidence we have suggests that these particular flow limited events may not have been clinically significant as they were not generally associated with microarousals. ${ }^{3}$ However, standard electroencephalographic techniques are not always able to detect microarousals which may be more obvious from the recording of cardiovascular sequelae. ${ }^{14}$ Thus, further data are required before the importance of this discrepancy is clear and at present the Autoset "apnoea" index should be interpreted with caution, especially as the study result is totally dependent on a single parameter - the flow signal (and its quality).

We chose to compare Autoset "apnoeas" with polysomnography apnoeas + hypopnoeas because the distinction between apnoeas and hypopnoeas is somewhat arbitrary ${ }^{15}$ and varies between centres. In addition, we suspect that there is inspiratory flow limitation during all clinically significant hypopnoeas and we therefore suspected, and the data show, that the Autoset device was picking up hypopnoeic events.

The Autoset is therefore dignostically useful and superior to oximetry alone. It has the additional advantage over most other limited sleep study equipment that it can also be used to determine the required pressure of continuous positive airway pressure (CPAP) therapy. We have estimated the cost of CPAP titration per patient to be $£ 35$ compared with $£ 154$ for polysomnography (including disposables, technician time for titration, night/patient, CPAP, education, and capital writedown based on 200 titrations per year).

Dr Douglas is an Advisor to ResCare Ltd. This study was not supported by ResCare Ltd.

1 Jennum P, Sjol A. Epidemiology of snoring in obstructive sleep apnoea in a Danish population age 30-60. Sleep Res 1992;1:240-4.

2 Young T, Palta M, Dempsey J, Skatrud J, Webber S, Bader $\mathrm{S}$. The occurrence of sleep-disordered breathing among middle aced adults. $N$ Engl $f$ Med 1993;328:1230-5.

3 Cheshire K, Engleman H, Deary I, Douglas NJ. Factors impairing daytime performance in patients with the sleep apnea/hypopnoea syndrome. Arch Intern Med 1992;152 $538-41$

4 Engleman HM, Martin SE, Deary IJ, Douglas NJ. Effect of continuous positive airway pressure treatment on daytime function in sleep apnoea/hypopnoea syndrome. Lance 1994;343:572-5.

5 Haraldsson P-O, Carenfelt C, Diderichsen F, Nygren A, Tingvall C. Clinical symptoms of sleep apnea syndrome and automobile accidents. ORL 1990;52:57-62.

6 Findley LJ, Umversat ME, Suratt PM. Automobile accidents involving patients with obstructive sleep apnea. Am Rev Respir Dis 1988;138:337-40.

7 Partinen M, Jamieson A, Guilleminault C. Long-term outcome for obstructive sleep apnea syndrome patients: mortality. Chest 1988;94:1200-4.

8 He J, Kryger MH, Zorick FJ, Conway W, Roth T. Mortality and apnea index in obstructive sleep apnea. Chest 1988 94:9-14.

9 Douglas NJ, Thomas S, Jan MA. Clinical value of polysomnography. Lancet 1992;339:347-50.

10 Berthon-Jones M. Feasibility of a self setting CPAP machine. Sleep 1993;16:S120-3.

11 Guilleminault C, Stoohs R, Klerk A, Cetal M, Maistros P. A cause of excessive daytime sleepiness: the upper airways A cause of excessive daytime sleepiness: the uppe

12 Johns MW. A new method for measuring daytime sleepiness: the Epworth sleepiness scale. Sleep 1991;14:540-5.

13 Bland JM, Altman DG. Statistical methods for assessing agreement between two methods of clinical measurement Lancet 1986;i:307-10.

14 Davies RJO, Vardivisy K, Clarke M, Stradling JR. Identification of sleep disruption and sleep disordered breathing from the systolic blood pressure profile. Thorax 1993 48:1242-7.

15 Gould GA, Whyte KF, Rhind GB, Airlie MAA, Catteral JR, Shapiro CM, et al. The sleep hypopnoea syndrome. Am Rev Respir Dis 1988;137:895-8. 